\title{
RESPOSTA DA PRÁTICA DE 12 SEMANAS DE NATAÇÃO SOBRE SAUDE MENTAL EM INDIVÍDUOS COM AMPUTAÇÃO DE MEMBROS
}

\author{
Beatriz Dieke Moreira \\ Universidade do Extremo Sul Catarinense \\ beatrizdieke@hotmail.com \\ Ramiro Doyenart \\ Universidade do Extremo Sul Catarinense \\ ramadoyenart@gmail.com
}

\author{
Karin Martins Gomes \\ Universidade do Extremo Sul Catarinense \\ karin@unesc.net \\ Luciano Acordi da Silva \\ Universidade do Extremo Sul Catarinense \\ luciano_acordi@unesc.net
}

\section{Resumo}

Esta pesquisa teve como objetivo verificar o efeito da prática de natação de 12 semanas sobre escores de saúde mental em indivíduos com amputação. Participaram do estudo 19 indivíduos entre 30 e 66 anos, separados em grupo 1: não amputados ( $\mathrm{n}=12)$ e grupo 2: amputados $(\mathrm{n}=7)$, ambos submetidos ao programa de natação com frequência de duas vezes por semana. Foram realizadas avaliação de depressão/ansiedade utilizando os inventários de Beck, e de autoestima utilizando o questionário de autoimagem/autoestima proposto por Steglich, antes e após o programa de natação utilizando o teste estatístico de Tukey. Os escores de ansiedade $(\mathrm{p}<0.05)$ de ambos os grupos apresentaram redução (não amputados:

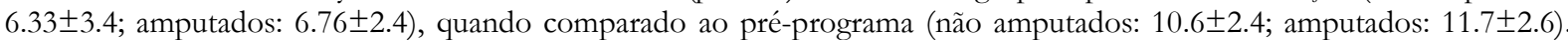
Em relação à autoestima, apenas o grupo de amputados teve aumento (181 \pm 8.6$)$ significativo $(\mathrm{p}<0.05)$, quando

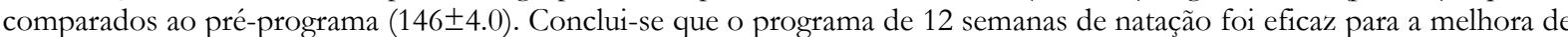
parâmetros de ansiedade e autoestima em pessoas com amputação.

Palavras-chave: Amputação. Natação. Saúde Mental.

\section{RESPONSE OF THE 12-WEEK PRACTICE OF SWIMMING ON MENTAL HEALTH IN INDIVIDUALS WITH LIMB AMPUTATION}

\begin{abstract}
This research aimed to verify the effect of 12 weeks swimming practice on mental health scores in individuals with amputation. Nineteen individuals with ages between 30 and 65 years old participated in the study, separated into group 1: no amputees $(n=12)$ and group 2: amputees $(n=7)$, both submitted to the swimming program twice a week. Self-esteem using the questionnaire of self-image and self-esteem of Steglich and depression/anxiety using the Beck Inventory were evaluated before and after the swimming program using Tukey's statistical test. In the anxiety scores $(\mathrm{p}<0.05)$ both groups

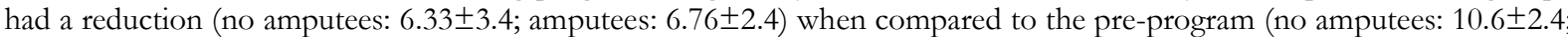
amputees: $11.7 \pm 2.6)$. Regarding self-esteem, only the group of amputees increased the scores (181 \pm 8.6 scores) significantly $(\mathrm{p}<0.05)$ when compared to the pre-program $(146 \pm 4.0)$. It was concluded that the 12 -week swimming program was effective in improving anxiety and self-esteem parameters in people with amputation.

Keywords: Amputation. Swimming. Mental Health.
\end{abstract}

\section{RESPUESTA DE 12 SEMANAS DE PRÁCTICA DE NATACIÓN SOBRE SALUD MENTAL EN INDIVIDUOS CON AMPUTACIÓN DE MIEMBROS}

\footnotetext{
Resumen

El objetivo de esta investigación fue verificar el efecto de 12 semanas de práctica de natación en la salud mental en individuos con amputación. Diecinueve individuos entre 30 y 66 años de edad participaron en el estudio, separados en grupo 1: no amputados ( $n=12$ ) y grupo 2: amputados $(n=7)$, ambos participaron, dos veces por semana, del programa de natación. A autoestima utilizando el cuestionario de autoimagen y autoestima de Steglich e depresión/ansiedad usando el Inventario de Beck fueron evaluadas antes y después del programa de natación usando la prueba estadística de Tukey. Las puntuaciones de ansiedad $(p<0,05)$ de ambos grupos se redujeron (sin amputados: 6,33 $\pm 3,4$; amputados: 6,76 $\pm 2,4)$ en

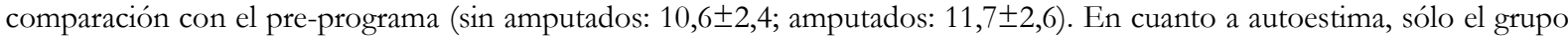
de amputados tuvo un aumento significativo $(181 \pm 8,6)(\mathrm{p}<0,05)$ en comparación con el pre-programa $(146 \pm 4,0)$. Se concluye que el programa de natación de 12 semanas fue efectivo para mejorar los parámetros de ansiedad y autoestima en personas con amputación.

Palabras clave: Amputación. Natación. Salud Mental.
} 
Resposta da prática de 12 semanas de natação sobre saúde mental em indivíduos com amputação de membros

\section{INTRODUÇÃO}

A literatura tem apontado a deficiência física como uma alteração que impossibilita ou prejudica o desempenho esperado da função de uma determinada parte do corpo afetada (DI NUBILA, 2010; WHO, 2002). Sendo assim, a amputação tem sido considerada como uma deficiência física (OMS, 2003). Mais especificamente, é o termo mais utilizado para definir a retirada parcial ou total de um membro do corpo, sendo este um método de tratamento e prevenção do agravamento de diversas doenças (OMS, 2003).

A amputação é classificada como uma deficiência física, que afeta pessoas de todas as idades, gêneros e condição social (DI NUBILA, 2010; OMS, 2003; WHO, 2002). Dados do IBGE apontam que aproximadamente $24 \%$ da população brasileira possui alguma deficiência, sendo que $7 \%$ dessa população apresenta deficiência motora, acometida por amputação, o que corresponde aproximadamente a três milhões de brasileiros (IBGE, 2013). A Constituição Federal e os princípios de diretrizes do Sistema Único de Saúde (SUS) redigiram decretos e leis de apoio à inclusão de pessoas com deficiência, no que tange à integração social, promoção da acessibilidade, manutenção da qualidade de vida e independência funcional (BRASIL, 1999; MINISTÉRIO DA SAÚDE, 2010).

Nesta direção, políticas públicas envolvendo o esporte como uma das ferramentas alternativas que ajudam na socialização e inclusão social de pessoas amputadas têm sido encorajadas (BRASIL, 2012; CASTRO; SOUZA, 2011; LUCCIA; SILVA, 2003; VIANNA; LOVISOLO, 2011). No campo educacional, a literatura tem apontado que o reconhecimento do esporte como ferramenta de socialização e inclusão social tem alavancado um crescente número de projetos esportivos destinados a pessoas portadoras de deficiência física (MINISTÉRIO DO ESPORTE, 2007; VIANNA; LOVISOLO, 2011). No Brasil, nos últimos anos, diversos projetos esportivos, como Esporte e Cidadania, Segundo Tempo e Brincando com Esporte, têm sido utilizados como complementares ao processo educacional, visando inclusão social e melhoria das condições de vida do deficiente físico (CARVALHO, 2003; CASTRO; SOUZA, 2011; KRAVCHYCHYN et al., 2019; MINISTÉRIO DO ESPORTE, 2007).

É fato que o esporte quando desenvolvido de maneira social e educacional atendendo à necessidade básica da sociedade no que compete respeito à individualidade e promoção de equidade provoca mudanças significativas na vida no ser humano com deficiência física, melhorando sua qualidade de vida (DANISH; NELLEN, 1997; TAFAREL, 2009; FEITOSA et al., 2017). Estudos têm reportado que o esporte melhora o desenvolvimento motor, a possibilidade de formação profissional, o desempenho intelectual (TAFAREL, 2009; FEITOSA 
Resposta da prática de 12 semanas de natação sobre saúde mental em indivíduos com amputação de membros

et al., 2017) a autossuperação, o autocontrole e a autoconfiança (KRAVCHYCHYN et al., 2019; VIANNA; LOVISOLO, 2011). O desenvolvimento de aspectos cognitivos como senso crítico, atenção e cultura também são apontados por alguns autores (CASTRO; SOUZA, 2011; VIANNA; LOVISOLO, 2011).

A prática de esportes vem sendo apontada como um dos tratamentos não medicamentosos que podem proporcionar benefícios relacionados à saúde mental, mais especificamente na depressão, ansiedade e autoestima (BAVOSO, 2018), por conta dos fatores fisiológicos e psicossociais (VIEIRA, 2009; MORAES, 2007). Em relação à pratica de natação para o deficiente, esta tem sido diferenciada de outros esportes, em virtude da liberdade de movimento e independência funcional que a mesma oferece (VIEIRA et al., 2018). Especificadamente nos indivíduos com amputação, alterações na saúde mental podem surgir por causa das diversas mudanças que a nova condição física implica (MILIOLI, 2012), e o esporte se apresenta como uma forma de prevenção, manutenção e tratamento da saúde mental de pessoas com deficiência física (MEDEIROS, 2018). É importante destacar a saúde mental como aspecto fundamental para a manutenção da saúde geral e bem-estar dos indivíduos, levando em conta que hoje se entende a saúde como um conjunto integrado das dimensões físicas, psicológicas e sociais. A saúde mental é mais do que a ausência de transtornos mentais, é uma construção diária dos aspectos psicológicos subjetivos, de relações interpessoais e mudanças sociais, essencial para todos os indivíduos (OPAS, 2016).

Dessa forma, este estudo teve por objetivo verificar o efeito da prática 12 semanas de natação sobre escores de saúde mental em indivíduos acometidos por amputação. Em relação à escolha da natação, esta se justifica em virtude que a mesma é realizada no meio aquático, possibilitando maior autonomia de movimentos sem a utilização de aparatos ortopédicos, uma vez que a água, através de suas propriedades como flutuação e empuxo, tem facilitado os movimentos corporais em pessoas portadoras de deficiência (VIEIRA et al., 2018).

\section{MATERIAIS E MÉTODOS}

O estudo foi desenvolvido pelos acadêmicos de Psicologia e Educação Física, bolsistas do Grupo de Pesquisa em Exercícios Aquáticos Avançados (GPEAA) vinculado ao Laboratório de Psicofisiologia do Exercício, aprovado no comitê de ética local (CAE 85652417.0.0000.0119). As aulas foram realizadas na piscina do complexo esportivo da Universidade do Extremo Sul Catarinense, Criciúma, Brasil. 
Resposta da prática de 12 semanas de natação sobre saúde mental em indivíduos com amputação de membros

Participaram do estudo 19 indivíduos de ambos os sexos, com idade entre 30 e 65 anos, separados em dois grupos: grupo 1: não amputados (n=12) (52 \pm 12 anos, $69 \pm 15 \mathrm{~kg}$ e $165 \pm 13 \mathrm{~cm})$, grupo 2: amputados ( $\mathrm{n}=7)(46 \pm 16$ anos, $72 \pm 14 \mathrm{~kg}$ e $169 \pm 10 \mathrm{~cm})$.

Em relação ao protocolo, os indivíduos foram submetidos a 12 semanas de aulas de natação, com frequência de duas vezes e tempo de sessão de 45 minutos. As aulas consistiram em atividades de adaptação aquática, exercícios de respiração, familiarização no ambiente aquático, nado crawl, atividades de relaxamento e descanso. A piscina do complexo esportivo da Unesc possui a metragem de $12,5 \mathrm{~m}$ x $25 \mathrm{~m}$, profundidade de $140 \mathrm{~cm}$ a $145 \mathrm{~cm}$, temperatura de $28 \mathrm{c}^{\circ}$ a $30 \mathrm{c}^{\circ}$ e tratamento com cloro. Quarenta e oito horas antes e após o programa de natação foram realizadas avaliação de depressão, ansiedade e autoestima.

Para avaliar a depressão, foi utilizado o Inventário de Depressão de Beck (BDI) (GORENSTEIN, 1998), validado no Brasil por Cunha (2001), composto por 21 itens sobre sintomas de depressão. Cada item possui quatro opões de respostas, variando a pontuação de $0 \mathrm{a}$ 3. É recomendado que a classificação seja referida como: sem depressão (menos de 10 pontos), depressão leve (entre 10 e 18 pontos), depressão moderada (entre 19 e 29 pontos) e depressão grave (entre 30 e 63 pontos).

Para avaliar a ansiedade, foi utilizado o Inventário de Ansiedade de Beck (BAI) (BECK et al., 1998; BAPTISTA; CARNEIRO, 2011), validado no Brasil por Cunha (2001), composto por 21 perguntas de autorrelato sobre intensidade de sintomas de ansiedade, contendo quatro opções de resposta, sendo elas absolutamente não; levemente; moderadamente e gravemente, com pontuação variando de 0 a 3 pontos. $O$ escore total varia de 0 a 63 , e permite a verificação do nível de intensidade da ansiedade. A classificação descrita no manual recomenda que o nível de ansiedade seja classificado em mínimo (0-7), leve (8-15), moderado (16-25) ou grave (26-63).

Para avaliar a autoestima, foi utilizado o questionário de autoimagem e autoestima de Steglich (VISCARDI; CORREIA, 2017), composto por 78 perguntas, divididas em quatro aspectos fundamentais (orgânico, social, intelectual e emocional). Cada pergunta do questionário possibilita cinco alternativas de respostas, organizadas em uma escala do tipo Likert (sim, quase sempre, várias vezes, algumas vezes e não), as quais são representadas por valores numéricos de 1 a 5. A soma das respostas fornece um escore para autoestima, variando de 41 a 205, e um escore para a autoimagem, oscilando de 37 a 185. Quanto mais alta a pontuação, mais altas são a autoimagem e a autoestima do indivíduo.

Para análise de dados, foi utilizado a ANOVA com teste estatístico de Tukey, e os dados foram analisados e expressos em média e desvio padrão, tendo $\mathrm{p}<0.05$ como valor de significância. 
Resposta da prática de 12 semanas de natação sobre saúde mental em indivíduos com amputação de membros

\section{RESULTADOS E ANÁLISES}

O presente estudo buscou avaliar o efeito da prática de natação de 12 semanas sobre sintomas de ansiedade, depressão e autoestima de saúde mental em indivíduos acometidos por amputação. Para tanto, foram realizadas avaliações 48 horas pré e após o programa de natação dos escores de depressão, ansiedade e autoestima em sujeitos não amputados e amputados.

A partir dos resultados obtidos neste estudo, foi possível observar melhora em alguns aspectos da saúde mental dos participantes, como sintomas de ansiedade e autoestima. Em relação aos sintomas depressivos, não apresentaram diferença significativa, quando comparado com os resultados obtidos na avaliação feita 48 horas antes do programa. Além disso, foi possível perceber a diferença dos resultados no grupo amputados e no grupo não amputados, indicando que a atividade física, mais especificamente a natação, pode exercer melhora nos aspectos da saúde mental desta população (amputados).

$\mathrm{Na}$ figura 1 são apresentados os dados referentes à avaliação de sintomas de depressão, pré e após programa de natação, no grupo não amputados e amputados. Os resultados apontam que os escores de depressão após o programa de natação de ambos os grupos (não amputados: $6.74 \pm 2.1$ escores; amputados: $6.55 \pm 2.5$ escores) não tiveram diferença significativa $(p>0.05) \mathrm{em}$ relação ao pré-programa (não amputados: 6.22 \pm 1.2 escores; amputados: $7.33 \pm 1.3$ escores).

Figura 1: Avaliação dos sintomas de depressão

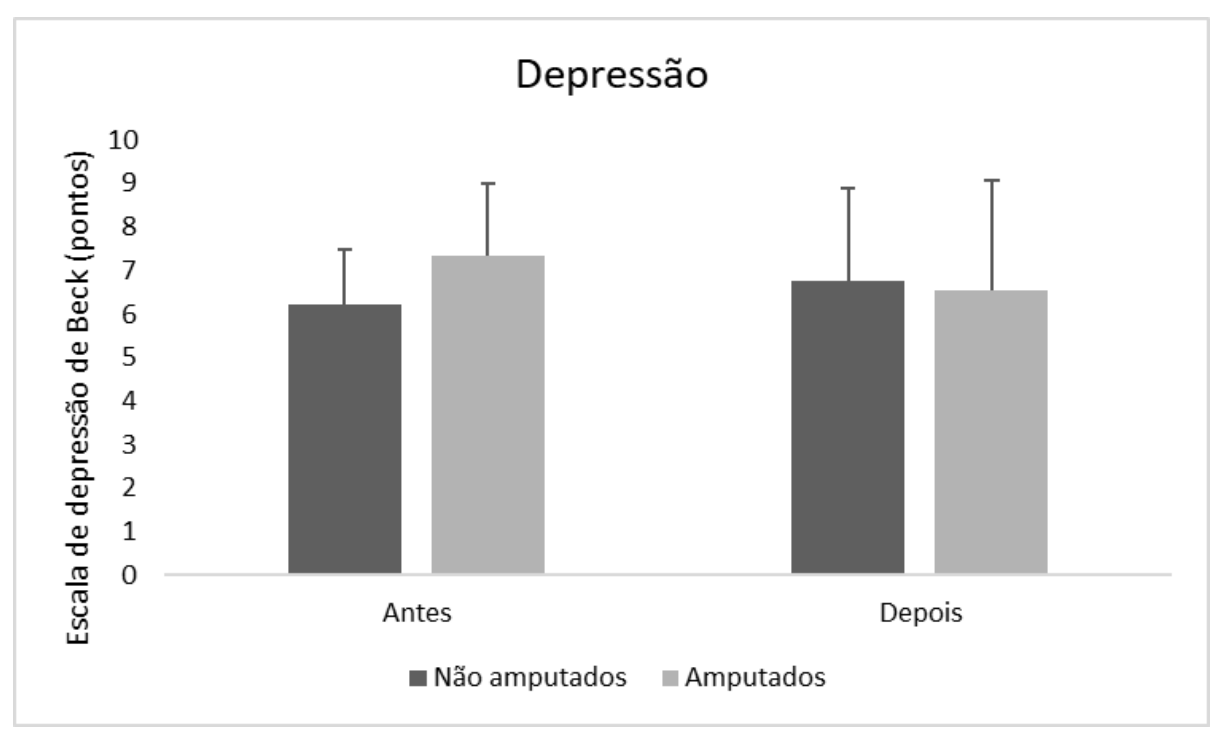

Fonte: Os autores.

A depressão tem sido definida como uma variedade de sintomas afetivos, cognitivos, psicóticos, biológicos, relativos à autovaloração, à vontade e à psicomotricidade, sendo suas 
Resposta da prática de 12 semanas de natação sobre saúde mental em indivíduos com amputação de membros

principais características o humor deprimido e a perda de interesse ou prazer (DALGALARRONDO, 2008; AMERICAN PSYCHIATRIC ASSOCIATION, 2014). Muitos estudos têm sinalizado para o aparecimento de depressão em indivíduos por conta de problemas sociais e financeiros, doenças psicossomáticas, excessiva cobrança da sociedade sobre felicidade e sucesso, doenças neurológicas, perdas, variação hormonal, entre outros (DALGALARRONDO, 2008; AMERICAN PSYCHIATRIC ASSOCIATION, 2014; CANTARELLI; SOARES; VOLPI, 2019 CASTRO, 2017). Além disso, os referidos estudos apontam também uma base biológica para a etiologia da depressão, baseada na deficiência da atividade dos neurotransmissores noradrenérgicos, dopaminérgicos e serotoninérgicos.

Indivíduos amputados às vezes apresentam sintomas depressivos ou piora na qualidade de vida após amputação de membros, por conta das implicações que esta nova condição pode trazer (MILIOLI, 2012). A amputação de membros interfere na imagem corporal e autoestima, autonomia funcional, adaptação à nova condição, percepção da sua posição e condição social, familiar, financeira e outros (MILIOLI, 2012). Nossos resultados apontam que ambos os sujeitos (amputados e não amputados), estão de acordo com a classificação, fora da faixa indicada de depressão (pré e pós programa). Psicologicamente, o exercício pode ajudar a reduzir a depressão por conta dos benefícios psicossociais e neurobiológicos que apresenta. A saúde mental está diretamente relacionada com o aspecto social (MILIOLI, 2012), e possibilitar a estes indivíduos um espaço para integração social é um fator que pode estar associado à melhora dos sintomas depressivos (MACEDO, 2008). De acordo com a visão psicológica da unidade corpo/mente, o trabalho somático (corpo) movimenta também o aspecto psíquico (saúde metal), ressaltando assim a importância da prática da atividade física (CASTRO, 2017). A literatura aponta também para um aumento na liberação das monoaminas, como dopamina, serotonina e noradrenalina, responsáveis pela regulação emocional (MORAES, 2007). Confirmando essas teorias, diversos estudos (BECERRA, et al., 2019 CASTRO, 2017; MORAES, 2007; NOCE; MELO, 2009) têm apontando que indivíduos depressivos, após sessões de exercícios físicos, apresentam melhora na saúde mental, integração social, adaptação a sua condição física e melhor qualidade de vida.

$\mathrm{Na}$ figura 2, são apresentados os dados referentes à avaliação de sintomas de ansiedade pré e após programa de natação. Os resultados apontam que os escores de ansiedade $(\mathrm{p}<0.05)$ após o programa de natação, de ambos os grupos (não amputados: 6.33 \pm 3.4 escores; amputados: $6.76 \pm 2.4$ escores), apresentaram diminuição quando comparados com o pré-programa (não amputados: 10.6 \pm 2.4 escores; amputados: $11.7 \pm 2.6$ escores), respectivamente. 
Resposta da prática de 12 semanas de natação sobre saúde mental em indivíduos com amputação de membros

Figura 2: Avaliação dos sintomas de depressão

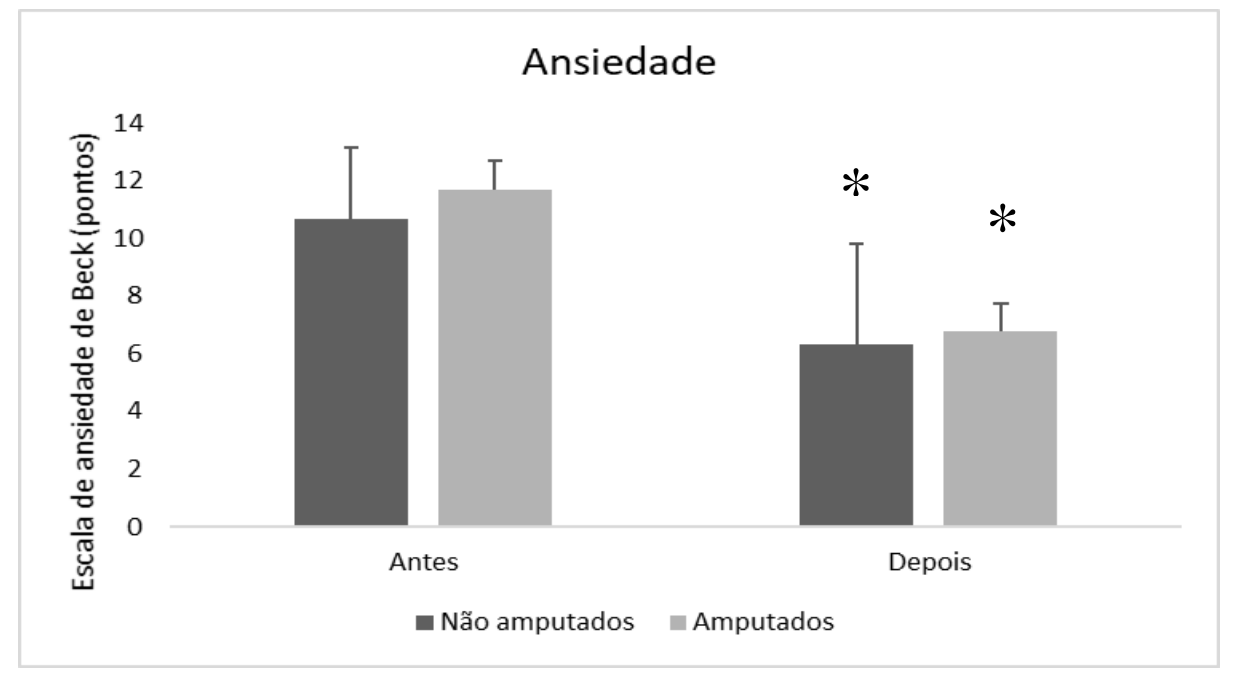

Fonte: Os autores.

A ansiedade tem sido definida como a antecipação de uma ameaça futura, caracterizada por aflição, angústia e diversos sintomas físicos e psíquicos, podendo se apresentar na forma de transtornos, como pânico, fobias, ansiedade generalizada e outros (DALGALARRONDO, 2008; AMERICAN PSYCHIATRIC ASSOCIATION, 2014). A ansiedade pode ainda ser diferenciada em estado e traço. A ansiedade estado refere-se a um momento emocional transitório, uma reação momentânea que provoca o aumento da atividade do sistema nervoso autônomo naquela situação, já a ansiedade traço refere-se a uma estrutura permanente, um modo habitual de reação, uma característica da personalidade do sujeito (CAPITAO; TELLO, 2004; DEKON, 2010; VERISSIMO et al., 2010).

Diversos estudos têm investigado o efeito da ansiedade após a prática de exercícios físicos. Nossos resultados apontam que em ambos os grupos (amputados e não amputados), os praticantes se beneficiaram da prática de natação e reduziram os escores de ansiedade. Os estudos mostram que a prática de exercício físico melhora aspectos de ansiedade em pessoas com deficiência física (MEDEIROS, 2018), e que a prática regular está associada à redução de índices de ansiedade e depressão, especialmente em exercícios aquáticos (ANTUNES, 2005; VIEIRA, 2009; LAMEGO, 2016; ARAUJO; MELLO; LEITE, 2007).

Em relação ao efeito agudo do exercício na ansiedade, Vieira et al. (2009) apontam que durante a prática do exercício ocorre a liberação de neurotransmissores como dopamina e bendorfina, responsáveis por proporcionar efeito tranquilizante e analgésico no pós-treino, percebido por meio da redução da frequência cardíaca, por exemplo. Já em relação ao efeito crônico do exercício na resposta ansiolítica, a literatura aponta que a prática regular pode resultar 
Resposta da prática de 12 semanas de natação sobre saúde mental em indivíduos com amputação de membros

na melhora de sintomas de tensão, depressão, raiva, fadiga e confusão, além de melhorar aspectos como agressividade, autoestima e interação social (VIEIRA, 2009). Psicologicamente, a prática de exercício proporciona ao indivíduo aumento da atividade social e relação interpessoal, o que gera aumento do bem-estar psicofisiológico e da qualidade de vida, principalmente quando aliado ao tratamento psicológico ou psiquiátrico (VIEIRA, 2009; ARAUJO; LEITE, 2007). A literatura aponta ainda que os efeitos positivos podem se dar por conta da distração dos estímulos desagradáveis durante a prática de exercício e o sentimento de autoconfiança que o desafio proporciona, bem como a própria interação social (MORGAN, 1985; NORTH, MCCULLAGH; TRAN, 1990; RANSFORD, 1982).

$\mathrm{Na}$ figura 3, são apresentados os dados referentes à avaliação da autoestima, pré e após programa de natação. Os resultados apontam que após o programa de natação, os escores de autoestima (não amputados: 173 \pm 4.0 escores; amputados: $181 \pm 8.6$ escores) não apresentaram resultados significativos para o grupo não amputados $(\mathrm{p}>0.05)$, porém apresentaram resultado significativo para o grupo amputados $(\mathrm{p}<0.05)$, em comparação com o pré-programa (não amputados: $169 \pm 6.0$ escores; amputados: $146 \pm 4.0$ escores).

Figura 3: Avaliação dos sintomas de autoestima

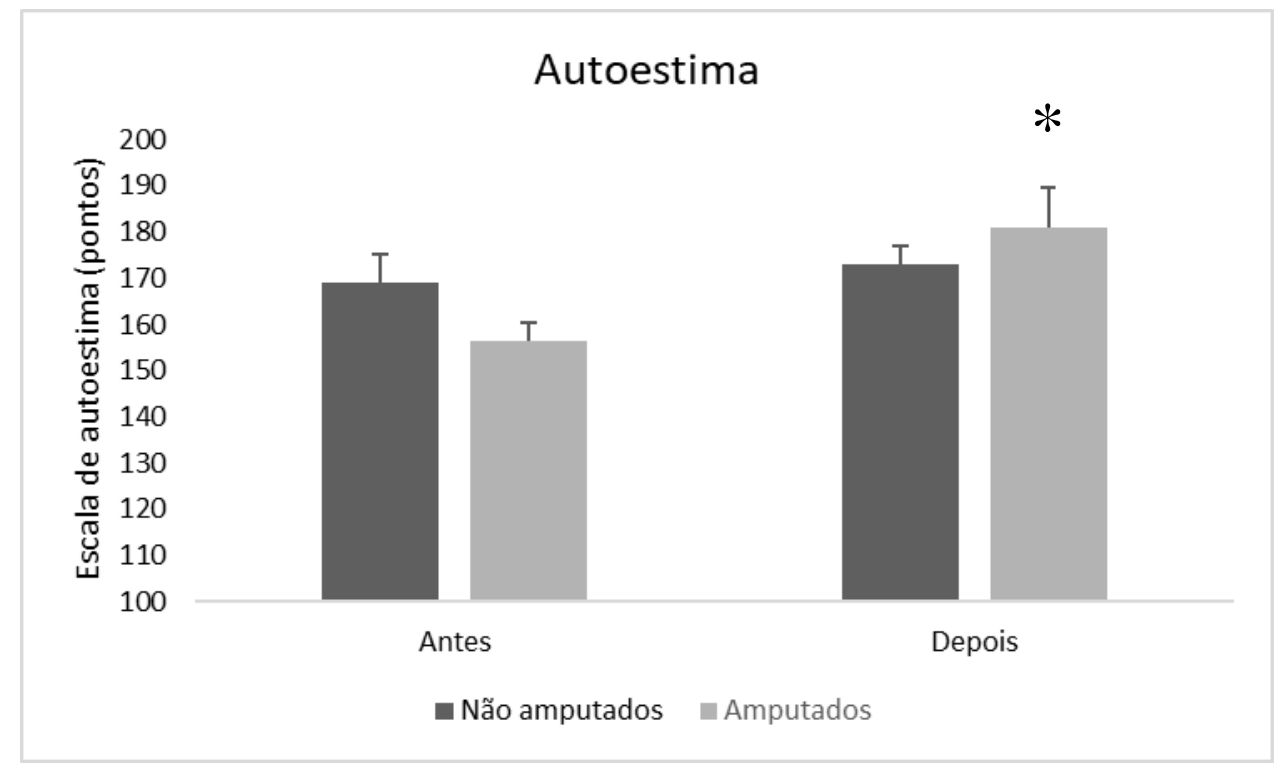

Fonte: Os autores.

A autoestima tem sido definida pela literatura como um conjunto de atitudes e sentimentos que o indivíduo tem de si mesmo, podendo ser positivos ou negativos (VISCARDI; CIRREIA, 2017; DEFFENDI; SCHELINI, 2014; SBICIGO; BANDEIRA, DELLAGLIO, 2010). De acordo com Deffendi e Schelini (2014), a autoestima é influenciada pelos 
Resposta da prática de 12 semanas de natação sobre saúde mental em indivíduos com amputação de membros

acontecimentos sociais, emocionais e psicofisiológicos, revelando qual o grau de importância, valorização e capacidade que o indivíduo tem de si mesmo. Nossos resultados apontam que, após o programa de natação, o grupo amputados obteve aumento da autoestima. Diversos estudos têm investigado o efeito do exercício na autoestima (MEURER, 2012; COSTA; SOARES; TEIXEIRA, 2007; ANTUNES; MAZO; BALBE, 2011; BAVOSO, 2018; MEURER; BENEDETTI; MAZO, 2009) e a literatura aponta que a prática de exercícios físicos está relacionada com o aumento do conceito de autoeficácia, autoimagem e melhora da aptidão e capacidade física, levando ao aumento da autoestima (ANTUNES; MAZO; BALBE, 2011; BAVOSO, 2018; MEURER; BENEDETTI; MAZO, 2009). Pesquisas também sugerem que idosos praticantes de atividades físicas apresentam menos autoconceitos e percepções negativas do que idosos não praticantes, visto que a autoestima está relacionada com sintomas depressivos (MEURER, 2012; ANTUNES; MAZO; BALBE, 2011; BAVOSO, 2018).

Em relação à pratica de natação nos deficientes, a literatura descreve que a mesma influencia diretamente nos aspectos fisiológicos e psicológicos dos mesmos, uma vez que os exercícios feitos na agua sofrem influência das propriedades físicas (densidade, pressão hidrostática e viscosidade), o que, por sua vez, tem efeito na musculatura e no aparelho locomotor através de uma maior irrigação sanguínea (SILVA et al., 2005; TSUTSUMI, 2004). Medeiros et al. (2018) demostraram que um programa de natação de 12 semanas reduziu o colesterol e melhorou a saúde mental em cadeirante. É fato que o ato de nadar propicia contração e relaxamento constante produzindo estímulos necessários para desenvolvimento muscular, melhora postural e ativação de neurotransmissores que ajudam a melhorar a saúde mental de pessoas com deficiência (MEDEIROS et al., 2018).

Como atividade de extensão, a presente pesquisa foi importante pois proporcionou aos bolsistas o desenvolvimento das habilidades e das competências profissionais, além de maior conhecimento prático e teórico sobre a área de atuação da Psicologia. Além disso, foi possível vivenciar e pôr em prática os conhecimentos adquiridos durante a graduação, bem como construir novos olhares para a realidade das pessoas com amputação e o trabalho que os profissionais de Psicologia e Educação Física podem proporcionar em conjunto. Uma das dificuldades do projeto foi a randomização dos participantes, já que poucas pessoas se disponibilizaram para participar do mesmo, alguns em função do tempo, outros por limitações pessoais. A acessibilidade da piscina foi outra limitação, dificultando o processo de entrada e saída da água. Por fim, o cuidado com o bem-estar físico e psicológico dos participantes foi intenso durante todo o processo de avaliação e participação das aulas. 
Resposta da prática de 12 semanas de natação sobre saúde mental em indivíduos com amputação de membros

\section{CONSIDERAÇÕES FINAIS}

Por meio deste estudo realizado no projeto de Extensão - Adversidades - e dos resultados obtidos a partir das avaliações pré e após o programa de natação, foi possível observar a melhora significativa dos aspectos da saúde mental, mais especificadamente na ansiedade e autoestima. Portanto, pode-se concluir que a atividade de extensão articulada com a pesquisa, envolvendo um programa de 12 semanas de natação, mostrou-se eficaz para a melhora dos parâmetros supracitados, em pessoas com amputação. A prática de esportes como forma de promoção de saúde mental tem se mostrado efetiva, principalmente por conta dos benefícios fisiológicos e psicossociais que proporciona aos praticantes, especialmente no que diz respeito aos sentimentos de superação relacionados aos desafios causados pela amputação.

Por fim, considera-se a importância da manutenção do programa de extensão e pesquisa, com o intuito de desenvolver práticas articuladas, pesquisas aplicadas à promoção de estilos de vida saudáveis para uma parcela da população que sofre por amputações, buscando avaliar este e outros meios de melhorar aspectos da saúde mental em pessoas com amputação por meio da atividade física aquática.

\section{FINANCIAMENTO}

O estudo foi financiado pelo Programa Diversidades, Inclusão e Direitos Humanos DIDH, no ano de 2017 e aprovado no comitê de ética local (CAE 85652417.0.0000.0119).

\section{REFERÊNCIAS}

AMERICAN PSYCHIATRIC ASSOCIATION et al. DSM-5: Manual diagnóstico e estatístico de transtornos mentais. Artmed Editora, 2014.

ANTUNES, Giselli; MAZO, Giovana Zarpellon; BALBÉ, Giovane Pereira. Relação da autoestima entre a percepção de saúde e aspectos sociodemográficos de idosos praticantes de exercício físico. Revista da Educação Física/UEM, v. 22, n. 4, p. 583-589, 2011.

ANTUNES, Hanna Karen Moreira et al. Depression, anxiety and quality of life scores in seniors after an endurance exercise program. Rev. Bras. Psiquiatr., São Paulo, v. 27, n. 4, p. 266271, Dec. 2005.

BAPTISTA, Makilim Nunes; CARNEIRO, Adriana Munhoz. Validade da escala de depressão: relação com ansiedade e stress laboral. Estudos de Psicologia (Campinas), v. 28, n. 3, p. 345-352, 2011. 
Resposta da prática de 12 semanas de natação sobre saúde mental em indivíduos com amputação de membros

BAVOSO, Daniel et al. Motivação e autoestima relacionada à prática de atividade física em adultos e idosos. Revista Brasileira de Psicologia do Esporte, v. 7, n. 2, 2018

BECERRA, et al. Percepção de atletas do rugby em cadeira de rodas sobre os apoios recebidos para a prática do esporte adaptado. Cadernos Brasileiros de Terapia Ocupacional, v. 27, n. 3, p. 615-627, 2019.

BECK A. T., EPSTEIN N., BROWN G., STEER R. A. An inventory for measuring clinical anxiety: psychometric properties. J Consult Clin Psychol. 1988;56(6):893-7.

BRASIL. Lei n. 3.298, 20 de dezembro de 1999. Política Nacional para a Integração da Pessoa Portadora de Deficiência [página na internet]. Brasília (DF). [acesso 2012 Mai 01]. Disponível em: http://portal. mec.gov.br/seesp/arquivos/pdf/dec3298.pdf

BRASIL. Lei n. LEI No 13.146, de 6 de julho de 2015. Institui a Lei Brasileira de Inclusão da Pessoa com Deficiência (Estatuto da Pessoa com Deficiência). [página na internet]. Brasília (DF). Acesso em 15 de 03 de 2020. Disponível em: http://www.planalto.gov.br/ccivil_03/_ato2015-2018/2015/lei/113146.htm

BRASIL. Ministério da Saúde. Secretaria de Atenção à Saúde. Departamento de Ações Programáticas Estratégicas. Diretrizes de atenção à pessoa amputada / Ministério da Saúde, Secretaria de Atenção à Saúde, Departamento de Ações Programáticas Estratégicas. - Brasília : Ministério da Saúde, 2012.

CANTARELLI, Estela Maris Lançoni; SOARES, Maria Márcia; VOLPI, José Henrique. O mal do século pela Psicologia Corporal. In: VOLPI, José Henrique; VOLPI, Sandra Mara (Org.) $24^{\circ}$ CONGRESSO BRASILEIRO DE PSICOTERAPIAS CORPORAIS. Anais. Curitiba: Centro Reichiano, 2019.

CAPITAO, Cláudio Garcia; TELLO, Renata Raveli. Traço e estado de ansiedade em mulheres obesas. Psicol. hosp. (São Paulo), São Paulo, v. 2, n. 2, dez. 2004 .

CARVALHO, J. Amputações de membros inferiores: em busca da plena reabilitação. São Paulo: Manole, 2003.

CASTRO, Gisele Fontenelle de Oliveira. Exercícios de alongamento e caminhada com idosos em uma UBS sob o enfoque da Psicologia Corporal. In: VOLPI, José Henrique; VOLPI, Sandra Mara (Org.) CONGRESSO BRASILEIRO DE PSICOTERAPIAS CORPORAIS, XXII, 2017. Anais. Curitiba: Centro Reichiano, 2017.

CASTRO, S. E SOUZA, D. Significados de um projeto social esportivo: um estudo a partir das perspectivas de profissionais, pais, crianças e adolescentes. Movimento. Porto Alegre, v. 17, n.04, p. 145-163, out/dez de 2011.

COSTA, Rudy Alves; SOARES, Hugo Leonardo Rodrigues; TEIXEIRA, José Antônio Caldas. Benefícios da atividade física e do exercício físico na depressão. Revista do Departamento de Psicologia. UFF, v. 19, n. 1, p. 273-274, 2007.

DALGALARRONDO, Paulo. Psicopatologia e semiologia dos transtornos mentais. Grupo AArtmed, 2008. 
Resposta da prática de 12 semanas de natação sobre saúde mental em indivíduos com amputação de membros

DANISH, S. J.; NELLEN, V. C. New roles for sport psychologists: teaching li skills through sport to at-risk youth. Quest, Champaign, v.49, p.100-13, 1997.

DEFFENDI, Luma Tiziotto; SCHELINI, Patrícia Waltz. Relação entre autoestima, nível intelectual geral e metacognição em adolescentes. Psicologia Escolar e Educacional, v. 18, n. 2, p. 313-320, 2014.

DEKON, Stefan Fiuza de Carvalho et al. Estudo comparativo entre índice anamnético de DTM e inventário de ansiedade traço-estado (IDATE). Jornal Brasileiro de Oclusão, ATM \& Dor Orofacial, v. 2, n. 7, 2010.

DI NUBILA, H. B. V. Nota Técnica - Uma introdução à CIF - Classificação Internacional de Funcionalidade, Incapacidade e Saúde. Revista Brasileira de Saúde Ocupacional, v. 35, n. 121, p. 122-123, 2010.

FEITOSA, Luzanira Correia et al. O efeito do esporte adaptado na qualidade de vida e no perfil biopsicossocial de crianças e adolescentes com paralisia cerebral. Revista Paulista de Pediatria, v. 35, n. 4, p. 429-435, 2017.

GORENSTEIN, Clarice; ANDRADE, L. H. S. G. Inventário de depressão de Beck: propriedades psicométricas da versão em português. Rev Psiq Clin, v. 25, n. 5, p. 245-50, 1998.

IBGE. Censo 2010 [página na internet]. 2013. Disponível em https:/ / censo2010.ibge.gov.br/ Acesso em 21/03/2020

KRAVCHYCHYN, Claudio et al. Projetos e programas sociais esportivos no Brasil: antecedentes históricos e reflexividade social. The Journal of the Latin American Socio-cultural Studies of Sport (ALESDE), v. 10, n. 1, p. 53-68, 2019.

LAMEGO, Murilo Khede et al. Aerobic exercise reduces anxiety symptoms and improves fitness in patients with panic disorder. MedicalExpress (São Paulo, online), São Paulo, v. 3, n. 3, M160306, June 2016.

LUCCIA, N.; SILVA, E. S. Aspectos técnicos de Amputações de Membros Inferiores. In: PITTA, G. (Ed.). Angiologia e cirurgia vascular: guia ilustrado. Rio de Janeiro: Guanabara Koogan, 2003.

MACEDO, Paula Costa Mosca. Deficiência física congênita e saúde mental. Revista da SBPH, v. 11, n. 2, p. 127-139, 2008.

MEDEIROS, Marina dos Santos de et al. Estudo de caso de um programa individualizado de natação em cadeirante portador de poliomielite: análise de parâmetros bioquímicos, qualidade de vida e capacidade física funcional. Revista Brasileira de Ciências do Esporte, v. 40, n. 1, p. 9499, 2018.

MEURER, Simone Teresinha; BENEDETTT, Tânia Rosane Bertoldo; MAZO, Giovana Zarpellon. Aspectos da autoimagem e autoestima de idosos ativos. Motriz. Journal of Physical Education. UNESP, p. 788-796, 2009. 
Resposta da prática de 12 semanas de natação sobre saúde mental em indivíduos com amputação de membros

MEURER, Simone Teresinha et al. Associação entre sintomas depressivos, motivação e autoestima de idosos praticantes de exercícios físicos. Revista Brasileira de ciências do esporte, v. 34, n. 3, p. 683-695, 2012.

MILIOLI, Renata et al. Qualidade de vida em pacientes submetidos à amputação. Revista de Enfermagem da UFSM, v. 2, n. 2, p. 311-319, 2012.

MINISTÉRIO DA SAÚDE (BR). Política Nacional de Saúde da Pessoa com Deficiência [página na internet]. Brasília (DF): MS; 2010 [acesso 2012 Mai01]. Disponível em: http:// bvsms.saude.gov.br/bvs/publicacoes/politica_nacional_pessoa_com_deficiencia.pdf

MINISTÉRIO DO ESPORTE. Programa Segundo Tempo: ação de funcionamento de núcleos. Brasília: UFRGS, 2007.

MORGAN, W. P. Affective beneficence of vigorous physical activity. Med Sci Sports Exerc. 1985;17:94-100. 66.

MORAES, Helena et al. O exercício físico no tratamento da depressão em idosos: revisão sistemática. Revista de psiquiatria do Rio Grande do Sul, v. 29, n. 1, p. 70-79, 2007.

NOCE, Franco; SIMIM, Mário Antônio de Moura; MELLO, Marco Túlio de. A percepção de qualidade de vida de pessoas portadoras de deficiência física pode ser influenciada pela prática de atividade física?. Revista Brasileira de Medicina do Esporte, v. 15, n. 3, p. 174-178, 2009.

NORTH, T. C., MCCULLAGH, P., TRAN, Z. V. Effect of exercise on depression. Exerc Sport Sci Rev. 1990;18:379-415. 67.

OMS. CIF: Classificação Internacional de Funcionalidade, Incapacidade e Saúde. São Paulo: EDUSP, 2003.

OPAS. OPAS/OMS apoia governos no objetivo de fortalecer e promover a saúde mental da população. 2016. Disponível em

https://www.paho.org/bra/index.php?option=com_content\&view =article\&id=5263:opas-omsapoia-governos-no-objetivo-de-fortalecer-e-promover-a-saude-mental-da-

populacao\&Itemid=839 Acesso em 07 de março de 2021.

RANSFORD, C. P. A role for amines in the antidepressant effect of exercise: a review. Med Sci Sports Exerc. 1982;4(1):1-10.

SBICIGO, Juliana Burges; BANDEIRA, Denise Ruschel; DELL'AGLIO, Débora Dalbosco. Escala de Autoestima de Rosenberg (EAR): validade fatorial e consistência interna. Psico-USF, v. 15, n. 3, p. 395-403, 2010.

SILVA, Maurício Corte Real da; OLIVEIRA, Ricardo Jacó de; CONCEIÇÃO, Maria Inês Gandolfo. Efeitos da natação sobre a independência funcional de pacientes com lesão medular. Revista Brasileira de Medicina do Esporte, v. 11, n. 4, p. 251-256, 2005.

TAFAREL, C. N. Z. Desporto educacional: realidade e possibilidades das políticas governamentais e das práticas pedagógicas nas escolas públicas. In: STIGGER, M.P.; LOVISOLO, H.R. (Orgs.) Esporte de rendimento e esporte na escola.Campinas: Autores Associados, 2009. p.71-102. 
Resposta da prática de 12 semanas de natação sobre saúde mental em indivíduos com amputação de membros

TSUTSUMI, Olívia et al. Os benefícios da natação adaptada em indivíduos com lesões neurológicas. Revista Neurociências, v. 12, n. 2, p. 82-86, 2004.

VERÍSSIMO, Sónia Margarida Alberto Correia et al. Relações entre ansiedade-estado e ansiedade-traço, sintomas depressivos e sensibilidade ao stresse em puérperas. 2010. Dissertação de Mestrado.

VIANNA, J. A., LOVISOLO, H. R. A inclusão social através do esporte: a percepção dos educadores. Rev. bras. Educ. Fís. Esporte, São Paulo, v.25, n.2, p.285-96, 2011.

VIEIRA, José Luiz Lopes et al. A prática da hidroginástica como tratamento complementar para pacientes com transtorno de ansiedade. Jornal Brasileiro de Psiquiatria, v. 58, n. 1, p. 8-16, 2009.

VIEIRA, Maressa Carvalho. A natação para a pessoa com deficiência: oferecimento e envolvimento de programas em Campinas e região. Conexões, v. 16, n. 2, p. 199-212, 2018.

VISCARDI, A. A. F.; CORREIA, P. M. S. Questionário de avaliação de autoestima e/ou de autoimagem: vantagens e desvantagens na utilização com idosos. R. bras. Qual. Vida, Ponta Grossa, v. 9, n. 3, p. 261-280, jul./set. 2017.

White RL, Babic MJ, Parker PD, Lubans DR, Astell-Burt T, Lonsdale C. Domain-Specific Physical Activity and Mental Health: A Meta-analysis. Am J Prev Med. 2017 May;52(5):653-666. doi: 10.1016/j.amepre.2016.12.008. Epub 2017 Jan 30. PMID: 28153647.

WHO. Towards a Common Language for Functioning, Disability and Health: ICF The International Classification of Functioning, Disability and Health. Genebra, 2002.

Recebido em: 28/09/2020

Aceito em: 20/07/2021 